\title{
Diagnosing pneumonia in patients with acute cough: clinical judgment compared to chest radiography
}

\author{
Saskia F. van Vugt ${ }^{1}$, Theo J.M. Verheij ${ }^{1}$, Pim A. de Jong², Chris C. Butler ${ }^{3}$, \\ Kerenza Hood ${ }^{3}$, Samuel Coenen ${ }^{4,5}$, Herman Goossens ${ }^{5}$, Paul Little ${ }^{6}$, and \\ Berna D.L. Broekhuizen ${ }^{1}$ on behalf of the GRACE Project Group
}

\begin{abstract}
Affiliations: ' Julius Center for Health, Sciences and Primary Care, University Medical Centre Utrecht, Utrecht, and ${ }^{2}$ Dept of Radiology, University Medical Centre Utrecht, Utrecht, The Netherlands. ${ }^{3}$ Institute of Primary Care and Public Health School of Medicine, Cardiff University, Cardiff, and ${ }^{6}$ Primary Care Medical Group, University of Southampton Medical School, Southampton, UK. ${ }^{4}$ Centre for General Practice, Vaccine and Infectious Disease Institute (VAXINFECTIO), University of Antwerp, Antwerp, and ${ }^{5}$ Laboratory of Medical Microbiology, VAXINFECTIO, University of Antwerp, Antwerp, Belgium.
\end{abstract}

Correspondence: S.F. van Vugt, University Medical Centre Utrecht, Julius Center for Health Sciences and Primary Care, Stratenum 5.149, PO Box 85500, 3508 GA Utrecht, The Netherlands.

E-mail: s.f.vanvugtQumcutrecht.nl

ABSTRACT Pneumonia is often diagnosed and treated empirically. We set out to determine the diagnostic accuracy of clinical judgment based on signs and symptoms to detect radiographic pneumonia in patients presenting with acute cough in primary care.

In 2810 European patients with acute cough, general practitioners (GPs) recorded whether they considered pneumonia to be present ("yes" or "no") immediately after history and physical examination. Chest radiography was performed within 1 week by local radiologists blind to other patient characteristics.

140 patients had radiographic pneumonia (5\%), of whom 41 (29\%) had been diagnosed as such. 31 (1\%) patients had a clinical diagnosis that was not confirmed by radiography $(n=2670)$. In clinically suspected pneumonia, $57 \%$ of subjects were subsequently diagnosed with radiographic pneumonia. Negative predictive value (NPV), sensitivity and specificity of GPs' clinical judgment were 96\%, 29\% and 99\%, respectively. Compared to patients with a clinical diagnosis of pneumonia, less severe symptoms were found in radiographic pneumonia cases not suspected clinically $(\mathrm{p}<0.05)$.

The predictive values of GPs' clinical judgment, particularly the high NPVs, are helpful in routine care. Nonetheless, the majority of diagnoses of radiographic pneumonias was not suspected on clinical grounds. There is a need to further support the detection of clinically relevant pneumonia in primary care.

@ERSpublications

There is a need to further support the detection of clinically relevant pneumonia in primary care http://ow.ly/mxw6Z

Earn CME accreditation by answering questions about this article. You will find these at the back of the printed copy of this issue or online at www.erj.ersjournals.com/misc/cmeinfo.xhtml

Received: July 182012 | Accepted after revision: Dec 03 2012 | First published online: Jan 242013

Support statement: This work was supported by the 6th Framework Programme of the European Commission (LSHMCT-2005-518226) and in Flanders, Belgium, this work was supported by the Research Foundation $(\mathrm{G} \cdot 0274 \cdot 08 \mathrm{~N})$.

Conflict of interest: Disclosures can be found alongside the online version of this article at www.erj.ersjournals.com

Copyright @ERS 2013 


\section{Introduction}

Acute cough and lower respiratory tract symptoms are among the most frequent reasons for seeking primary healthcare. Most patients presenting with these symptoms in primary care are diagnosed as having acute bronchitis and a minority as having pneumonia. In general, pneumonia requires antibiotic treatment with close monitoring, while acute bronchitis is self-limiting and does not benefit meaningfully from antibiotic treatment [1-3]. General practitioners (GPs) usually have to either diagnose or exclude pneumonia on the basis of history, symptoms and signs alone, as it is neither feasible nor appropriate to perform chest radiographs in all patients presenting with acute cough or lower respiratory tract symptoms. Initial decisions on the most likely diagnosis, treatment and additional testing are therefore most often based on GPs' clinical judgment.

However, the performance of this clinical judgment is largely unknown. Several primary care studies have assessed the diagnostic value of signs and symptoms as individual items or in combination for diagnosing pneumonia $[4,5]$, but there is limited data on the accuracy of GPs' clinical judgment, independent of a formal diagnostic model in lower respiratory tract infection (LRTI). MACFARLANE $e$ al. [1] found that GPs could not identify the need for antibiotic treatment with sufficient precision in patients with LRTI who had microbiological evidence of a bacterial infection. MeLBYe et al. [5] compared GPs' clinical diagnosis of pneumonia with findings on chest radiographs and found that GPs made a clinical diagnosis of pneumonia in only a minority of the patients who had a radiographic diagnosis of pneumonia. However, these studies are limited by the small number of cases of pneumonia they included.

Knowledge on the performance of an empirical diagnosis of pneumonia in routine general practice is important for the development and implementation of diagnostic tools, such as formal diagnostic models and near-patient tests. We therefore set out to assess the diagnostic accuracy of a purely clinical diagnosis of pneumonia with a radiographic finding of pneumonia as the reference standard in a large sample of patients presenting with acute cough in primary care.

\section{Methods}

\section{Design and study population}

294 GPs in 16 primary care research networks in 12 European countries included 3106 consecutive patients presenting with acute cough in the GRACE-09 study (Genomics to combat Resistance against Antibiotics in Community-acquired LRTI in Europe; www.grace-lrti.org) between October 2007 and April 2010. Recruited networks had access to a minimum of 20000 patients and had a track record of conducting research. A national network coordinator and a national network facilitator took responsibility for their network's set up, recruitment and data management. Eligible patients were $\geqslant 18$ years old, with an acute or worsened cough ( $\leqslant 28$-day duration) as the main symptom or any clinical presentation considered to be caused by LRTI by the GP, and consulting for the first time for this illness episode. Exclusion criteria were pregnancy, breastfeeding and any serious condition associated with impaired immunity. All participants gave written, informed consent and the medical ethics committees of the participating centres approved the study.

\section{GPs' assessment and radiographic findings}

GPs recorded patients' symptoms, signs, comorbidities (diabetes and respiratory and cardiovascular disease), medication use and, directly thereafter, his or her estimated diagnosis (open question) at presentation. All patients underwent chest radiography in local services within 1 week. Radiographs were assessed by local service radiologists blind to patients' current clinical information, but aware of the studies inclusion criteria and with free access to previous radiographs if available. The radiologists made a radiographic diagnosis by selecting one of the following fixed option responses: "normal X-ray", "acute bronchitis", "lobar or bronchopneumonia" or "other". There was a free text option to specify "other". Radiologists were allowed to inform responsible GPs if they found consolidation or made any diagnosis that required further investigation. In all other cases, GPs received the results after the study had been completed.

A subset of 1544 chest radiographs that were available for reassessment were reassessed by an independent radiologist (P.A. de Jong) at the University Medical Center Utrecht (Utrecht, the Netherlands) and interobserver variability was determined [6-8].

\section{Data analysis}

Of the patients' clinical characteristics, $<1 \%$ were missing. Missing data rarely occur completely at random and, therefore, imputation using multiple regression techniques was applied as recommended by many methodologists $[9,10]$.

GPs' empirical diagnoses were categorised into: "pneumonia", "exacerbation chronic obstructive pulmonary disease (COPD)/asthma", "upper respiratory tract infection (URTI)", "LRTI" (either 
unspecified or viral cause), "acute cough" or "other", and the proportions of these diagnoses in each country were calculated.

We subsequently dichotomised GPs' diagnoses into "pneumonia" or "no pneumonia" and calculated sensitivity, specificity, positive predictive value (PPV), negative predictive value (NPV) and likelihood ratios (LR) of the clinical assessment of the presence or absence of pneumonia.

Because radiographic pneumonia was diagnosed less often than expected in some networks, based on previous prevalence studies $[1,11]$, we repeated the analyses excluding all countries with pneumonia prevalence $<3 \%$ (Poland, France and Italy).

We compared patients with radiographic pneumonia who had been diagnosed with pneumonia on clinical grounds with those with radiographic pneumonia but who had not received this diagnosis on empirical grounds in terms of symptoms, signs and patients' severity scores.

Data were analysed using SPSS (version 17.0; SPSS Inc., Chicago, IL, USA).

\section{Results}

\section{Patient characteristics}

Of the 3106 eligible patients with acute cough, 296 were excluded because chest radiography was not performed $(n=258)$ or was of insufficient quality $(n=28)$, and 10 because results of GPs' clinical judgment were not available (fig. 1). The mean age of the 2810 remaining patients was 50 years, $40 \%$ were male and $28 \%$ were current smokers (table 1). 140 (5\%) patients had radiographic pneumonia. The observed proportional agreement between the original and second radiological diagnosis was 1445 (94\%) in 1544 patients. Kappa was 0.45 (95\% CI 0.36-0.54: moderate agreement). The observed positive agreement (48\%) was much lower than the negative agreement (97\%). Patient characteristics did not differ significantly between analysed patients and excluded patients (due to missing radiograph results or clinical judgment), apart from age. Excluded patients were younger (mean age 44 years) (results not shown).

\section{GPs' diagnoses}

$72(3 \%)$ patients were judged to have pneumonia by the GPs. The proportion of diagnosed COPD/asthma exacerbations was $4 \%$, of URTI $16 \%$ and of acute bronchitis $73 \%$, which included $61 \%$ "not otherwise specified" and $12 \%$ specified as a viral cause. $4 \%$ of the patients were diagnosed with a symptom and not a disorder ("acute cough"), and three patients with "other diagnoses" (headache, cardiac failure or pertussis). The proportion of the most common diagnoses differed between the 12 European countries (table 2). 91\% of patients underwent chest radiography within 5 days and the mean \pm SD duration between the first consultation for acute cough and chest radiography was $1.6 \pm 2.6$ days.

\section{GPs' clinical judgment of pneumonia}

The GPs' judgment regarding the presence or absence of pneumonia agreed with the radiographic diagnosis in 2680 (95\%) patients (table 3). Of all patients diagnosed with radiographic pneumonia, 29\% were diagnosed with pneumonia by GPs after history and physical examination (sensitivity). Specificity, PPV and NPV were 99\%, 57\% and 96\%, respectively. Positive and negative LRs for GPs' clinical judgment were 24 and 0.72 , respectively. The agreement between clinical and radiographic diagnoses after exclusion of networks based in Poland, France and Italy is also shown in table 3, and this resulted in a sensitivity, specificity, PPV and NPV of 30\%, 99\%, 59\% and $96 \%$, respectively.

Patients included

Patients with acute cough included $n=3106$

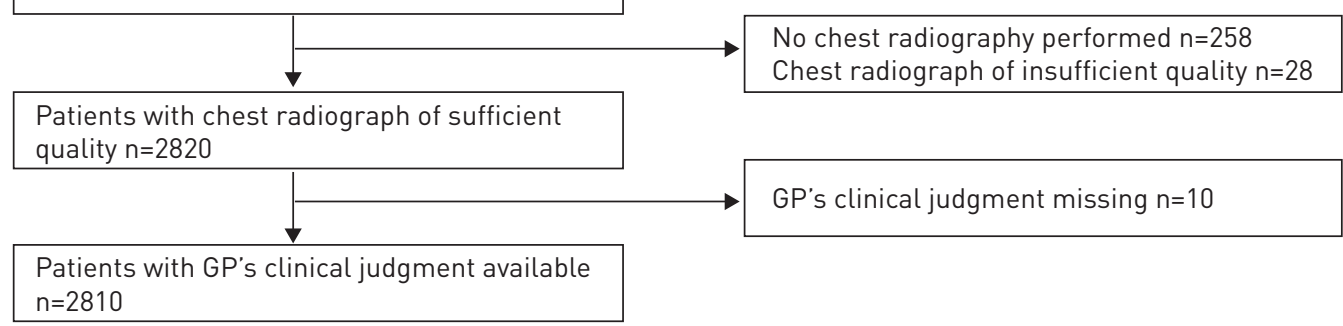

FIGURE 1 Flow chart of the study and participants. GP: general practitioner. 
TABLE 1 Patient characteristics, including symptoms and signs, in 2810 patients presenting with acute cough in primary care

$\begin{array}{lc}\text { Patient history } & 50 \pm 17 \\ \text { Age years } & 1122(40) \\ \text { Male } & 780(28) \\ \text { Current smoking } & 763(27) \\ \text { Comorbidity (pulmonary, cardiac or DM) } & 9 \pm 8 \\ \text { Days ill prior to consultation } & 2808(100) \\ \text { Cough present } & 1592(57) \\ \text { Breathlessness present } & 1301(46) \\ \text { Chest pain present } & \\ \text { Physical examination } & 358(13) \\ \text { Diminished vesicular breathing } & 265(9) \\ \text { Crackles } & 121(4) \\ \text { Temperature }>37.8^{\circ} \mathrm{C} & 72(3) \\ \text { Clinical judgment of general practitioner } & \\ \text { Pneumonia present } & 140(5) \\ \text { Chest radiograph (reference test) } & \end{array}$

Data are presented as mean \pm SD or $\mathrm{n}(\%)$. DM: diabetes mellitus. ${ }^{\#}$ : pulmonary comorbidity was defined as history of asthma or chronic obstructive pulmonary disease and cardiac comorbidity was defined as history of heart failure or ischaemic heart disease.

\section{Clinical importance of clinically unidentified radiographic pneumonia}

Patients who had not received a clinical diagnosis of pneumonia but who had radiographic pneumonia $(\mathrm{n}=99)$, had lower self-reported symptom severity scores and less severe individual symptoms, such as fever, crackles and signs of systemic inflammatory response syndrome, when compared with those patients who had both an empirical clinical diagnosis and radiographic diagnosis of pneumonia $(\mathrm{n}=41)($ table 4$)$.

\section{Discussion}

\section{Main findings}

In patients presenting with acute cough in primary care, GPs diagnosed pneumonia on clinical grounds alone in $29 \%$ of all patients who were subsequently found to have radiographic pneumonia (positive LR 24). In patients without radiographic pneumonia $(n=2670)$, GPs diagnosed pneumonia in $31(1 \%)$ patients on clinical grounds alone. The NPV of the GPs' judgment was $96 \%$.

\section{Strengths and limitations}

To our knowledge, this is the first study on GPs' diagnostic judgment of pneumonia in acute cough with an adequate number of pneumonia cases. Strengths of our study include the recording of clinical judgment in

TABLE 2 General practitioners' diagnoses in 2810 patients presenting with acute cough, by country

\begin{tabular}{|c|c|c|c|c|c|c|c|c|c|}
\hline \multirow[t]{2}{*}{ Country } & \multirow[t]{2}{*}{ Patients $\mathbf{n}$} & \multicolumn{7}{|c|}{ Diagnosis } & \multirow{2}{*}{$\begin{array}{c}\text { Radiographic } \\
\text { pneumonia }\end{array}$} \\
\hline & & Pneumonia & $\begin{array}{l}\text { Exacerbation } \\
\text { COPD/asthma }\end{array}$ & $\begin{array}{c}\text { LRTI } \\
\text { unspecified }\end{array}$ & LRTI viral & Acute cough & URTI & Other & \\
\hline Belgium & 367 & 2.5 & 5.4 & 67.3 & 2.2 & 0.3 & 0.3 & 0.0 & 5.4 \\
\hline France & 29 & 0.0 & 3.4 & 62.1 & 10.3 & 3.4 & 3.4 & 0.0 & 0.0 \\
\hline Germany & 158 & 4.4 & 7.0 & 41.1 & 32.9 & 11.4 & 11.4 & 1.0 & 5.1 \\
\hline Italy & 76 & 1.3 & 1.3 & 96.1 & 0.0 & 0.0 & 0.0 & 0.0 & 2.6 \\
\hline The Netherlands & 273 & 4.4 & 5.5 & 38.8 & 15.0 & 0.4 & 0.4 & 0.0 & 8.1 \\
\hline Poland & 551 & 0.5 & 1.8 & 61.2 & 14.7 & 2.7 & 2.7 & 0.0 & 0.7 \\
\hline Slovakia & 146 & 0.0 & 2.1 & 78.8 & 0.7 & 0.0 & 0.0 & 0.0 & 4.1 \\
\hline Slovenia & 74 & 0.0 & 0.0 & 86.5 & 13.5 & 0.0 & 0.0 & 0.0 & 5.4 \\
\hline Spain & 585 & 5.6 & 6.2 & 72.0 & 6.7 & 5.6 & 5.6 & 0.0 & 6.5 \\
\hline Sweden & 98 & 2.0 & 2 & 74.5 & 19.4 & 1.0 & 1.0 & 0.0 & 6.1 \\
\hline England & 203 & 2.0 & 5.4 & 49.3 & 24.6 & 1.5 & 1.5 & 0.0 & 6.9 \\
\hline Wales & 250 & 0.4 & 2.4 & 40.0 & 10.4 & 16.4 & 16.4 & 0.0 & 6.4 \\
\hline Total & & 2.6 & 4.1 & 61.2 & 11.7 & 4.1 & 4.1 & 0.1 & 5.0 \\
\hline
\end{tabular}

Data are presented as \%, unless otherwise stated. COPD: chronic obstructive pulmonary disease; LRTI: lower respiratory tract infection; URTI: upper respiratory tract infection. 


\begin{tabular}{|c|c|c|c|}
\hline \multirow[t]{2}{*}{ GPs' judgment } & \multicolumn{3}{|c|}{ Chest radiography } \\
\hline & Pneumonia present & Pneumonia absent & Total \\
\hline \multicolumn{4}{|l|}{ Including all countries } \\
\hline Pneumonia present & 41 & 31 & 72 \\
\hline Pneumonia absent & 99 & 2639 & 2738 \\
\hline Total & 140 & 2670 & 2810 \\
\hline \multicolumn{4}{|l|}{$\begin{array}{l}\text { Without including low- } \\
\text { prevalence countries }\end{array}$} \\
\hline Pneumonia present & 40 & 28 & 68 \\
\hline Pneumonia absent & 94 & 1992 & 2086 \\
\hline Total & 134 & 2020 & 2154 \\
\hline
\end{tabular}

Data are presented as $n$.

conditions similar to those of routine care, immediately after taking a history and performing a physical examination, and all patients undergoing a similar reference test for pneumonia, irrespective of the GPs' clinical judgment. A limitation regarding the reference standard is that all chest radiographs were examined by local radiologists in the European centres. We attempted to increase uniformity in assessment by using a protocol for reporting. Interobserver variability remained, but the moderate unweighted kappa of 0.45 was similar to other studies $[6,8]$. Moreover, re-analysis with radiographic pneumonia as a reference test, as diagnosed by a second single assessor, showed comparable results (sensitivity, specificity, PPV and NPV of $20 \%, 98 \%, 42 \%$ and $94 \%$, respectively).

A second possible limitation of our study regards the fact that, given that LRTI is common, many more eligible patients than were recruited would have been consulted during the recruitment period. Therefore, we probably did not achieve the goal of recruiting consecutive, eligible patients (e.g. the networks based in France, Slovakia and Slovenia included no patients with a clinical diagnosis of pneumonia). Nevertheless,

\section{TABLE 4 Characteristics of clinically identified and unrecognised radiographic pneumonias}

\begin{tabular}{|c|c|c|c|}
\hline Clinical characteristics & $\begin{array}{l}\text { Clinically identified } \\
\text { radiographic pneumonia }\end{array}$ & $\begin{array}{l}\text { Clinically unrecognised } \\
\text { radiographic pneumonia }\end{array}$ & p-value \\
\hline \multicolumn{4}{|l|}{ Symptoms and signs } \\
\hline Runny nose & 44 & 64 & 0.031 \\
\hline Fever & 90 & 41 & $<0.001$ \\
\hline Chest pain & 68 & 52 & 0.068 \\
\hline Comorbidity (pulmonary, cardiac or DM) & 20 & 23 & 0.629 \\
\hline Abnormal auscultation lungs & 83 & 50 & $<0.001$ \\
\hline Diminished vesicular breathing & 15 & 20 & 0.441 \\
\hline Crackles & 66 & 16 & $<0.001$ \\
\hline Rhonchi & 27 & 23 & 0.652 \\
\hline Heart rate $>100$ beats $\cdot \mathrm{min}^{-1}$ & 24 & 7 & 0.004 \\
\hline Breathing frequency $>24$ breaths $\cdot \mathrm{min}^{-1}$ & 10 & 2 & 0.040 \\
\hline Blood pressure $<90 / 60 \mathrm{mmHg}$ & 12 & 4 & 0.073 \\
\hline \multicolumn{4}{|l|}{ Gradation of illness } \\
\hline Severe cough & 30 & 43 & 0.143 \\
\hline Severe breathlessness & 26 & 13 & 0.133 \\
\hline Severe fever & 31 & 7 & 0.002 \\
\hline Severe chest pain & 23 & 8 & 0.045 \\
\hline Severe general unwellness & 25 & 26 & 0.912 \\
\hline Severe interference with daily activities & 23 & 27 & 0.697 \\
\hline
\end{tabular}

Data are presented as \%, unless otherwise stated. DM: diabetes mellitus. ${ }^{\#}$ : pulmonary comorbidity was defined as history of asthma or chronic obstructive pulmonary disease and cardiac comorbidity was defined as history of heart failure or ischaemic heart disease; ${ }^{\uparrow}$ : as judged by the patient. 
we believe the findings of the study are at low risk of selection bias because feedback from recruiting clinicians during and after the study indicated that the time required to recruit and assess each patient made sequential recruitment of every eligible patient impossible. A recent observational study [12] in the same recruiting primary care networks used the same case definition and a very similar baseline case record form, but was much simpler to implement and that study recruited patients with similar characteristics to the current study (e.g. age 45 years, $37 \%$ male, and 26\% comorbidity of either pulmonary, cardiac or diabetes mellitus).

\section{Comparison with other studies}

Several previous studies in primary care assessed the diagnostic value of history taking and physical examination to identify pneumonia $[4,5]$. A validation study of clinical prediction models in primary care patients found PPVs ranging from 17 to $47 \%$ and NPVs ranging from $79 \%$ to $88 \%$ [11], but did not include GPs' clinical judgment. Two previous studies evaluated GPs' empirical clinical judgments in primary care patients with LRTI. MACFARLANE et al. [1] assessed GPs' clinical judgment in 318 adults with LRTI, and found no relationship between their clinical judgment and microbiological evidence of bacterial and/or atypical pathogens]. MELBYE et al. [5] studied 402 adults with LRTI, of whom 20 had pneumonia on chest radiography, and found that GPs identified seven of the 20 cases with radiographic pneumonia on clinical grounds. They also diagnosed pneumonia in 22 patients without radiographic pneumonia. Our results confirm that GPs make a clinical judgment of pneumonia in the minority of patients who are subsequently found to be diagnosed with pneumonia on chest radiography.

\section{Implication of the results}

It has been shown that many clinicians justify their use of broad-spectrum antibiotics on the grounds of not wanting to miss a case of pneumonia [13]. It is therefore helpful that we found the NPV of clinical judgment to be as high as $96 \%$. This can support GPs to rely on their ability to exclude pneumonia and withhold antibiotic treatment in a large proportion of their patients [12].

Still, the large proportion of radiographic pneumonia not diagnosed on clinical grounds alone may seem worrisome. However, radiographic pneumonia cases that were clinically unrecognised in our study had symptoms of severe illness (e.g. fever and chest pain) less often at presentation (table 4). It has been shown before that milder pneumonia cases not suspected clinically may have a relatively more benign course [14]. Moreover, we assume that the serious negative health effects of a "missed" pneumonia in outpatients can be limited by instructing patients to revisit if symptoms persist or worsen. Improving the NPV of GPs' assessment of $96 \%$ will be difficult. However, two small studies showed that adding C-reactive protein level to signs and symptoms can result in a NPV of $97 \%[4,15]$. Further studies on the diagnostic value of C-reactive protein point-of-care tests in primary care in larger cohorts are needed.

\section{Conclusions}

Although the predictive values of GPs' clinical judgment are helpful in daily management of patients and especially the NPV of the clinical assessment is high, the majority of radiographic pneumonias were not recognised on clinical grounds alone. There is a need to further support the detection of clinically relevant pneumonia in primary care.

\section{Acknowledgements}

We would like to thank the entire GRACE team for their diligence, expertise and enthusiasm. Finally, we are indebted to all of the patients who consented to be part of GRACE, without whom this study would not have been possible.

\section{References}

1 Macfarlane J, Holmes W, Gard P, et al. Prospective study of the incidence, aetiology and outcome of adult lower respiratory tract illness in the community. Thorax 2001; 56: 109-114.

2 Smith SM, Fahey T, Smucny J, et al. Antibiotics for acute bronchitis. Cochrane Database Systematic Rev 2004; 4: CD000245.

3 Gonzales R, Steiner JF, Sande MA. Antibiotic prescribing for adults with colds, upper respiratory tract infections, and bronchitis by ambulatory care physicians. JAMA 1997; 278: 901-904.

4 Hopstaken RM, Muris JW, Knottnerus JA, et al. Contributions of symptoms, signs, erythrocyte sedimentation rate, and C-reactive protein to a diagnosis of pneumonia in acute lower respiratory tract infection. Br J Gen Pract 2003; 53: $358-364$

5 Melbye H, Straume B, Aasebo U, et al. Diagnosis of pneumonia in adults in general practice. Relative importance of typical symptoms and abnormal chest signs evaluated against a radiographic reference standard. Scand J Prim Health Care 1992; 10: 226-233.

6 Hopstaken RM, Witbraad T, van Engelshoven JM, et al. Inter-observer variation in the interpretation of chest radiographs for pneumonia in community-acquired lower respiratory tract infections. Clin Radiol 2004; 59: 743-752.

7 Boersma WG, Daniels JM, Löwenberg A, et al. Reliability of radiographic findings and the relation to etiologic agents in community-acquired pneumonia. Respir Med 2006; 100: 926-932. 
8 Albaum MN, Hill LC, Murphy M, et al. Interobserverreliability of the chest radiograph in community-acquired pneumonia. PORT Investigators. Chest 1996; 110: 343-350.

9 Donders AR, van der Heijden GJMG, Stijnen T, et al. Review: a gentle introduction to imputation of missing values. J Clin Epidemiol 2006; 59: 1087-1091.

10 Mackinnon A. The use and reporting of multiple imputation in medical research - a review. J Intern Med 2010; 268: 586-593.

11 Graffelman AW, le Cessie S, Knuistingh NA, et al. Can history and exam alone reliably predict pneumonia? J Fam Pract 2007; 56: 465-470.

12 Butler CC, Hood K, Verheij T, et al. Variation in antibiotic prescribing and its impact on recovery in patients with acute cough in primary care: prospective study in 13 countries. BMJ 2009; 338: b2242.

13 Wood F, Simpson S, Butler CC. Socially responsible antibiotic choices in primary care: a qualitative study of GPs' decisions to prescribe broad-spectrum and fluroquinolone antibiotics. Fam Pract 2007; 24: 427-434.

14 Lim WS, Macfarlane JT. Importance of severity of illness assessment in management of lower respiratory infections. Curr Opin Infect Dis 2004; 17: 121-125.

15 Khalil A, Kelen G, Rothman RE. A simple screening tool for identification of community-acquired pneumonia in an inner city emergency department. Emerg Med J 2007; 24: 336-338. 